Florian Kock

e-mail: florian.kock@dlr.de

\author{
Cornelius Ferrari \\ e-mail: cornelius.ferrari@dlr.de
}

German Aerospace Center (DLR), Institute of Vehicle Concepts (FK),

Stuttgart D-70569, Germany

\section{Flatness-Based High Frequency Control of a Hydraulic Actuator}

This paper presents the design and implementation of a nonlinear feedforward control algorithm for a hydraulic actuator driven by a multistage servo valve. Combined with a conventional feedback control algorithm, high frequencies can be achieved even for largescale strokes. In addition to the desired trajectory, the feedforward controller accepts the predicted dynamic load on the hydraulic actuator as an input. The performance of the control concept as well as the advantages of the load input are verified in simulations and experiments. Being exemplarily used for realizing a crankshaft-less test stand for free piston engines, the control algorithm is potentially suitable for further applications using hydraulic actuators in high frequency domain. [DOI: 10.1115/1.4005047]

\section{Introduction}

At the German Aerospace Center (Deutsches Zentrum für Luftund Raumfahrt e.V., DLR), a free piston internal combustion engine (ICE) is being developed. It is designed to convert chemical energy of a fuel into electrical energy. It can be utilized as a range extender in future electric vehicles. The engine consists of three main components:

(1) A combustion section converting chemical energy into kinetic energy.

(2) A linear generator converting kinetic energy into electric energy.

(3) A gas spring storing energy and inverting the piston movement.

The combustion piston, generator rotor, and gas spring piston are rigidly coupled and perform a transversal oscillation between bottom dead center and top dead center. Compared to conventional combustion engines, this engine concept leads to fully flexible operating parameters such as variable stroke and variable compression ratio. Moreover, the shape of the piston trajectory is variable and in general different from the kinematically determined trajectory of a crank drive. For more information about the project, see Ref. [1].

The development of the free piston engine requires an approach, where every component is tested separately at first. Bringing all three components into service at the same time is an impossible task, because each of them is characterized by a large set of parameters which influence each other. In order to be able to test every single component, it is necessary to use an actuator, which replaces the missing components and, therefore, drives the piston. The actuator moves each component along a predefined desired trajectory so that it can be operated independently from the other two components. Pursuing this strategy, the components can be brought into service and adjusted separately before putting them together. A hydraulic linear actuator is chosen for this application as it is able to generate high forces and it allows to adapt the trajectories without any mechanical changes. Figure 1 shows the hydraulic engine test stand with all three components attached to the hydraulic actuator.

Challenging demands are made on the additional actuator. It is supposed to operate at high frequencies $(30 \mathrm{~Hz})$ with large strokes $(90 \mathrm{~mm})$ and high forces (even the inertia forces add up to $10 \mathrm{kN}$ ). Each of these values had been reached separately in the past, but no application is known justifying the need to combine them in a

Contributed by the Dynamic Systems Division of ASME for publication in the Journal of Dynamic Systems, Measurement, and Control. Manuscript received September 7, 2010; final manuscript received July 19, 2011; published online December 29, 2011. Assoc. Editor: Nariman Sepehri. single operating point. The aforementioned values are higher than the ones being necessary for handling test rigs in automotive suspension development. For a summary of the state of the art in this field, see Deuschl [2]. The highest velocity recorded by Deuschl is $2.5 \mathrm{~m} / \mathrm{s}$, whereas the combined demands for the engine test stand are equivalent to an average velocity of $4.2 \mathrm{~m} / \mathrm{s}$ and a maximum velocity of $8.5 \mathrm{~m} / \mathrm{s}$. In earthquake simulations [3], shaking tables are used to reaching strokes of $300 \mathrm{~mm}$ and maximum frequencies of $100 \mathrm{~Hz}$ but not in a single operating point. The limiting factor for shaking tables is the maximum acceleration of $7 \mathrm{~g}$ [3], while the accelerations demanded for the free piston linear generator (FPLG) test stand reach up to $160 \mathrm{~g}$. Moreover, the requirements concerning the frequency bandwidth include the fundamental frequency of up to $30 \mathrm{~Hz}$ as well as three to seven multiples of it, because the desired plunger motion is a nonharmonic periodic signal.

To improve the performance of the system, the hardware setup is chosen as follows. The main component is a double-action hydraulic working piston, customized with an aluminum plunger in order to reduce the moving mass. It is driven by a three-stage proportional servo valve with a nominal flow rate of $1000 \mathrm{l} / \mathrm{min}$. For an overview of the hydraulic system specifications, see Table 1. A high precision incremental displacement measurement system detects the plunger position. For the control task, a digital controller is implemented and run on a dSPACE real-time system.

The design and implementation of the plunger position controller will be discussed in this paper. Section 2 describes the control task as well as the plant and its mathematical model. Controller design is outlined in Secs. 3 and 4 (feedforward and feedback controller, respectively). In Sec. 5, measurement results are presented and a conclusion is made.

\section{Mathematical Modeling and Control Task}

In order to develop flatness-based feedforward controller, a model of the plant is built and implemented in MATLAB/SIMULINK. The hydraulic cylinder is described by its motion equation (Eq. (1)) and the differential equation of pressure generation for each chamber (found on the basis of Ref. [4] and exemplified for chamber $\mathrm{A}$ in Eq. (2))

$$
\begin{gathered}
m_{p} \cdot \ddot{x}_{p}=\left(p_{A}-p_{B}\right) \cdot A_{p}-d_{p} \dot{x}_{p}+F_{\text {Load }} \\
\dot{p}_{A}=\frac{E_{\mathrm{oil}}}{V_{0}+A_{p} x_{p}}\left(\dot{Q}_{A}-k_{l i p}\left(p_{A}-p_{B}\right)-k_{l i v} \dot{x}_{p}-A_{p} \dot{x}_{p}\right)
\end{gathered}
$$

Therein, $p_{A}$ and $p_{B}$ are the oil pressures in the two chambers, $x_{p}$ is the plunger position of the hydraulic actuator, $A_{p}$ is the effective area of the plunger, and $\dot{Q}_{A}$ is the total volume flow into chamber A. $F_{\text {Load }}$ is the force that is applied to the plunger by the three 


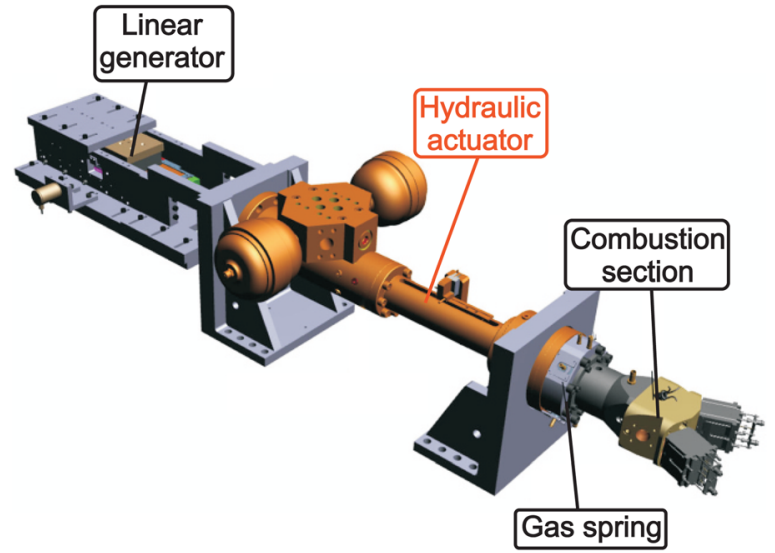

Fig. 1 Hydraulic actuator on the test stand

components of the FPLG and can be regarded as a disturbance for the position controller discussed in this paper.

Internal leakage (leakage between the two chambers) consists of a pressure proportional and a velocity proportional component. This leakage model was originally developed within a swashplate pump model and afterward adapted for hydraulic working cylinders [5].

The parameters $k_{l i p}$ and $k_{l i v}$ for the leakage model are listed in Table 2 among other model parameters. The parameters for leakage and friction can neither be measured directly nor are they available in any data sheets. In order to find appropriate values, the model is used as a part of the feedforward controller (see Sec. 3) and the model parameters are tuned such that the controller performance is satisfactory. Experimental results show that the entire plant model is comparatively insensitive to changes in those parameters, so that the effort for finding the values can be kept low. Nevertheless, the more accurate treatment of parameters and the robustness against dynamic changes of them are starting points for further performance optimizations, for example, by integrating quantitative feedback laws (QFT, [6,7]). These enhancements would be important especially for applying the model to plants with more significant friction or leakage effects.

Figure 2 shows the hydraulic working cylinder connected to the main valve stage. The main valve stage is the third stage of the entire servo valve. The first two stages (not shown in the figure) are significantly smaller and are used to set the valve slide position $x_{v}$ of the main valve slide. Depending on this position, four orifices (one for each of the flows $\dot{Q}_{0 \rightarrow A}, \dot{Q}_{A \rightarrow T}, \dot{Q}_{0 \rightarrow B}, \dot{Q}_{B \rightarrow T}$ ) are continuously opened or closed in order to regulate the volume flow toward the working cylinder. Moving the valve slide in positive direction of $x_{v}$ leads to an increase of $\dot{Q}_{0 \rightarrow A}$ and $\dot{Q}_{B \rightarrow T}$, so that in total, chamber A is filled with oil and chamber B is discharged. In the state shown in the figure, the flows $\dot{Q}_{0 \rightarrow B}$ and $\dot{Q}_{A \rightarrow T}$ are zero because the associated orifices are completely

Table 1 Hydraulic system specifications

\begin{tabular}{lcc}
\hline \hline \multicolumn{1}{c}{ System specification } & Value & Unit \\
\hline Actuator manufacturer & Hänchen & - \\
Actuator type & Servofloat 328 & - \\
Plunger diameter & 70 & $\mathrm{~mm}$ \\
Plunger rod diameter & 50 & $\mathrm{~mm}$ \\
Maximum stroke & 96 & $\mathrm{~mm}$ \\
Moving mass & 3.2 & $\mathrm{~kg}$ \\
Hydraulic natural frequency & 546 & $\mathrm{~Hz}$ \\
Hydraulic supply pressure & 290 & $\mathrm{bar}$ \\
Valve manufacturer & Moog & - \\
Valve type & $\mathrm{D}-792$ & - \\
Nominal flow rate & 1000 & $1 / \mathrm{min}$ \\
\hline \hline
\end{tabular}

Table 2 Model parameters

\begin{tabular}{lcc}
\hline \hline Parameter & Value & Unit \\
\hline$A_{p}$ & 18.85 & $\mathrm{~cm}^{2}$ \\
$m_{p}$ & $3.2+$ workload & $\mathrm{kg}$ \\
$d_{p}$ & $2 \cdot 10^{3}$ & $\frac{\mathrm{Ns}}{\mathrm{m}}$ \\
$E_{\text {oil }}$ & $1.5 \cdot 10^{9}$ & $\frac{\mathrm{N}}{\mathrm{m}^{2}}$ \\
$k_{\text {lip }}$ & $1.2 \cdot 10^{-10}$ & $\mathrm{M}^{2}$ \\
$k_{l i v}$ & $2.4 \cdot 10^{-4}$ & $\mathrm{~m}$ \\
$V_{0}$ & 8.5 & $\overline{\mathrm{Ns}}$ \\
$r_{v}$ & 15 & $\mathrm{~cm}^{3}$ \\
$\alpha$ & 0.49 & $\mathrm{~mm}$ \\
\hline \hline
\end{tabular}

closed. Moving the valve slide to a negative position opens the two last-mentioned orifices and closes the other two.

The model of the servo valve consists of two parts. First, a model of the main valve stage, which directly regulates the mass flow toward the hydraulic cylinder, is developed from Bernoulli's orifice equation [4]. Depending on the position of the valve slide, the output ports of the valve are connected either with the pressure supply or with the tank (containing hydraulic fluid at environmental pressure). As a result, two different equations are generated and a distinction of cases becomes necessary. Therefore, the flow rate function is characterized not only by nonlinearity but also by a discontinuity of the derivative of the flow rate at the neutral position of the valve slide

$$
\dot{Q}_{A}= \begin{cases}\dot{Q}_{0 \rightarrow A}=\alpha \cdot 2 \pi \cdot r_{v} \cdot x_{v} \cdot \sqrt{\frac{2}{\rho}} \cdot \sqrt{p_{0}-p_{A}} & \text { if } \quad x_{v} \geq 0 \\ -\dot{Q}_{A \rightarrow T}=\alpha \cdot 2 \pi \cdot r_{v} \cdot x_{v} \cdot \sqrt{\frac{2}{\rho}} \cdot \sqrt{p_{A}} & \text { if } \quad x_{v} 0\end{cases}
$$

Second, a black box model is developed in order to represent the transfer behavior between the valve's input signal $u_{v}$ and the position of the main valve slide $x_{v}$. The position $x_{v}$ of the valve slide depends on the position of the valve slides of the first two stages. The three-stage valve is equipped with an internal controller. The aim of the valve-internal controller is to generate a proportional (" $P$ ") relation between the input voltage $u_{v}$ and the valve slide position $x_{v}$. As neither the mechanical configuration of these valve stages nor the control algorithm and parameters are documented precisely, forming an accurate model based on physical laws is not feasible. Instead, this part of the plant is represented by a black box model. This nonlinear transfer behavior has been identified by

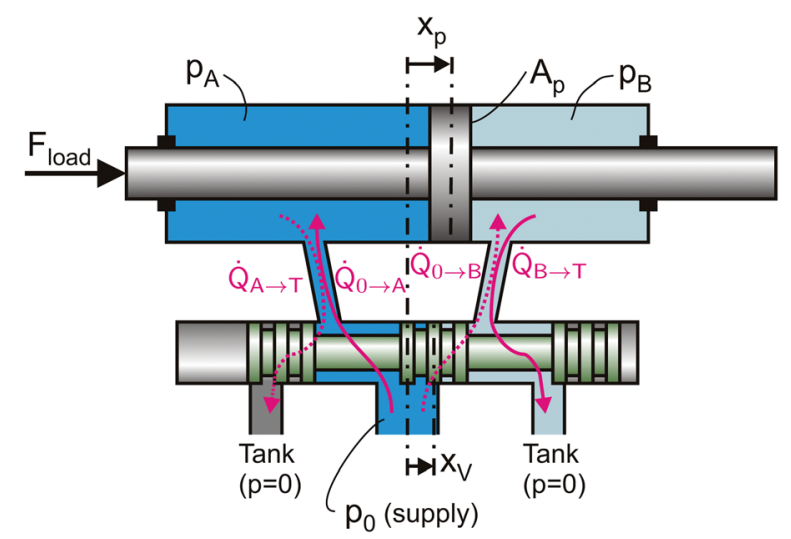

Fig. 2 Hydraulic working piston plunger and last stage of servo valve 


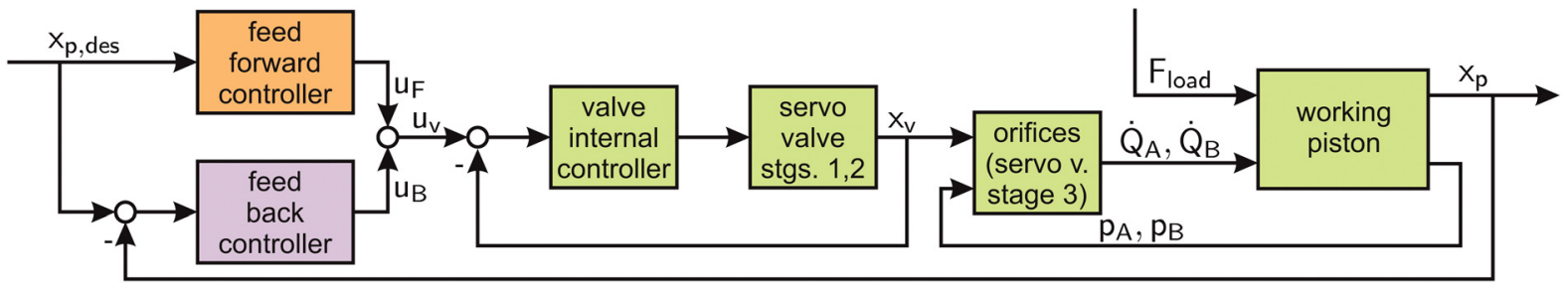

Fig. 3 Cascaded control circuit

the valve manufacturer. In order to invert the model with manageable algebraic effort, a linear transfer function is required to represent this section of the plant. The linearization is possible in two different levels of accuracy.

System identification based on step response data leads to the conclusion that the amplitude response is most suitably described by a fourth order delay element in combination with a dead time element. This is the more accurate linearization, in the following referred to as $P T_{4} T_{d}$. The system description can be simplified by reducing the transfer behavior to a single dead time element (with a longer dead time constant than the dead time element in the $P T_{4} T_{d}$ system). In the following, this less accurately linearized system is referred to as $P T_{d}$. The following steps can be applied to both levels of model detail. Although the procedure of inverting the system is the same in both cases, the resulting equations become by far more unhandy in case of the $P T_{4} T_{d}$. As this paper treats the system theory and control design rather than algebraic aspects, the rest of the paper refers to the $P T_{d}$ system.

In addition to the valve-internal controller, which controls the valve slide position, another controller is required to track the desired trajectory of the plunger. This leads to a cascaded structure, which is shown in Fig. 3. The outer loop controller is the subject of this paper. The control signal is the voltage $u_{v}$, and the set point is the desired periodic trajectory.

\section{Feedforward Control Design}

The comparatively slow dynamics of the hydraulic valve lead to a low phase margin in the desired frequency range. Thus, it is impossible to control the system only by a feedback controller; a

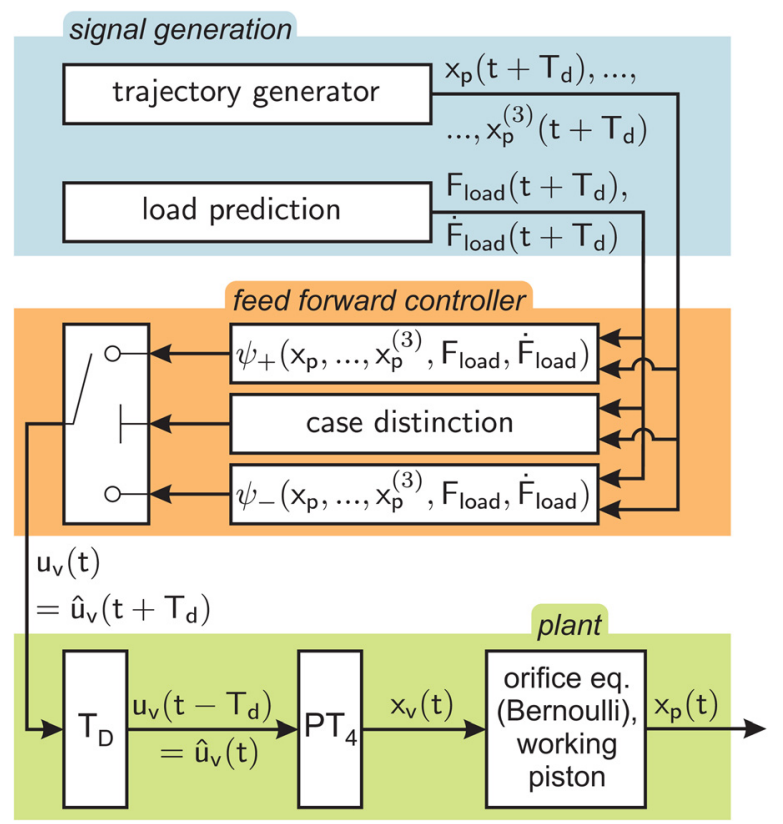

Fig. 4 Plant with signal generator and feedforward controller consisting of inverse model and case distinction feedforward controller becomes necessary. Since the feedforward controller is based on a simplified model and disturbances occur, a feedback controller is needed in addition. Thus, to solve the control task of following a desired trajectory of the plunger position, a combination of both feedforward and feedback controller is used. A similar control structure for hydraulic plants has been used for different applications with larger time constants in the past $[8,9]$.

The feedforward controller is designed using a model-based approach. The result of the system inversion will be an algorithm calculating the valve input voltage $u_{v}(t)$ that is necessary to track a desired trajectory $x_{p}(t)$ of the plunger. Therefore, the model derived in Sec. 2 of this paper has to be inverted. Discontinuity, dead time, and nonlinearity have to be treated within the procedure of system inversion. Especially, nonlinearity is an aspect that is part of a wide range of control problems $[10,11]$.

In a first step, the system is inverted only for $x_{v} \geq 0$ (limited operating range in order to avoid the discontinuity). Inverting the dead time is avoided by introducing a time-shifted input voltage

$$
\hat{u}_{v}(t)=u_{v}\left(t-T_{d}\right)
$$

$T_{d}$ is the dead time given by the $P T_{d}$ model of the valve dynamics. Some minor simplifications are made concerning the bulk modulus of the oil in the chambers and the pressure drop at the orifices. The stiffness of the oil and the surrounding walls, pipes, and hosing is assumed to be independent of the plunger position. The pressure drop throughout the valve is assumed to be equally distributed to both orifices which are open at the same time (see Fig. 2 ). The result is a continuous and causal, yet nonlinear system of differential equations. The number of states is reduced to four (Eqs. (5)-(10))

$$
\begin{gathered}
x=\left[\begin{array}{l}
x_{1} \\
x_{2} \\
x_{3} \\
x_{4}
\end{array}\right]=\left[\begin{array}{c}
x_{p} \\
\dot{x}_{p} \\
p_{A}-p_{B} \\
x_{v}
\end{array}\right], \quad u=\left[\begin{array}{l}
u_{1} \\
u_{2}
\end{array}\right]=\left[\begin{array}{c}
\hat{u}_{v} \\
F_{\text {Load }}
\end{array}\right] \\
\dot{x}_{1}=x_{2} \\
\dot{x}_{2}=\frac{x_{3} A_{p}-d_{p} x_{2}+u_{2}}{m_{p}} \\
\dot{x_{3}}=2 \frac{E_{\text {oil }}}{V_{0}} \cdot\left(\dot{Q}-k_{\text {lip }} x_{3}-k_{\text {liv }} x_{2}-A_{p} x_{2}\right) \\
x_{4}=k_{v} u_{1} \\
\alpha=\left\{\begin{array}{c}
\alpha \cdot 2 \pi \cdot r_{v} \cdot x_{4} \cdot \sqrt{\frac{p_{0}-x_{3}}{\rho_{\text {oil }}}} \text { if } \quad x_{v} \geq 0 \\
\alpha \cdot 2 \pi \cdot r_{v} \cdot x_{4} \cdot \sqrt{\frac{p_{0}+x_{3}}{\rho_{\text {oil }}}} \text { if } x_{v}
\end{array}\right.
\end{gathered}
$$

Implemented in a simulation environment like MATLAB/SIMULINK, this set of equations can be used to simulate the system's reaction $x$ to a given set of input values $u$. Finding $u$ for a desired trajectory $x_{p}$ is more complex, as the system (Eqs. (5)-(10)) cannot simply be solved for $u$. Instead, the system has to be inverted.

For inverting nonlinear systems, Fliess et al. introduced the concept of flatness in 1992 [12]. It can be shown that the system (Eqs. (5)-(10)) is differentially flat with the flat output $y=\left[\begin{array}{ll}x_{1} & u_{2}\end{array}\right]^{T}$, 


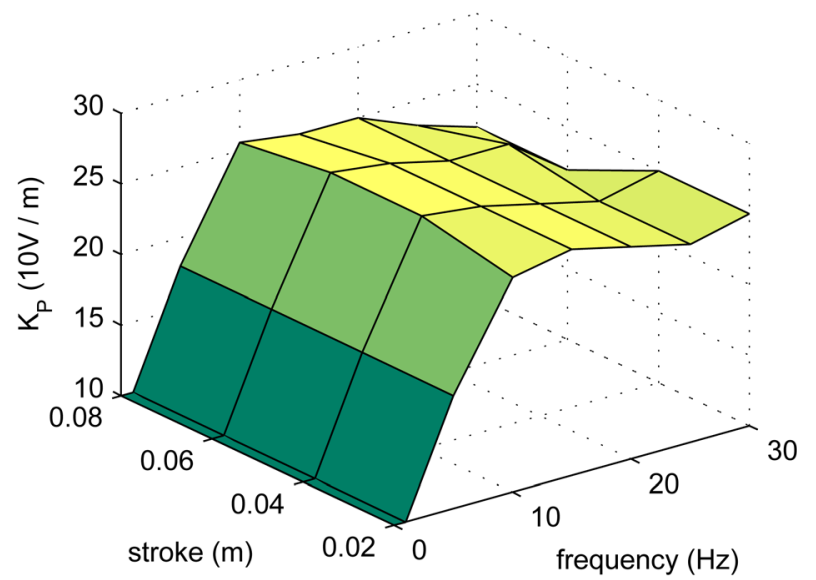

Fig. 5 Feedback controller variable gain $K_{P}$

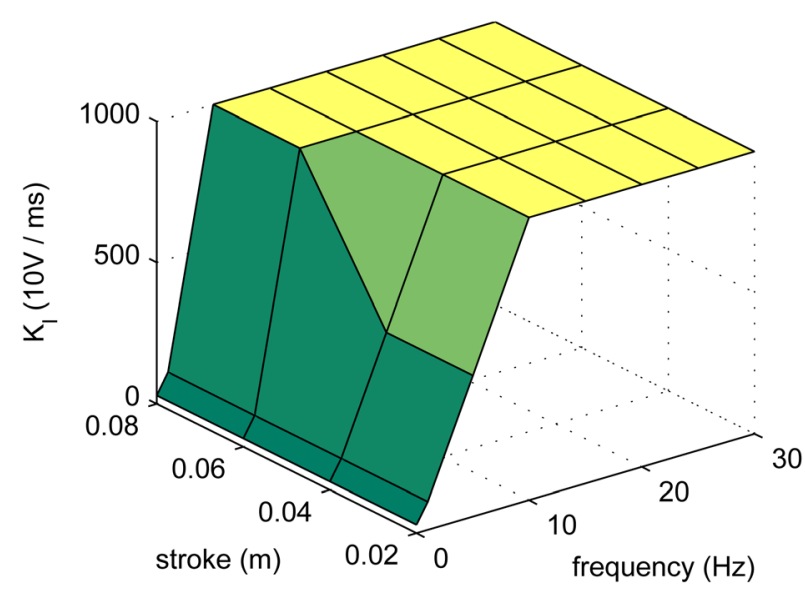

Fig. 6 Feedback controller variable gain $K_{l}$

where $x_{1}$ is the trajectory and $u_{2}$ is the load force. The (forward) model of the plant is regarded as a multiple input single output (MISO) system with the two inputs control voltage $u_{v}$ and load force $F_{\text {Load. }}$. For this reason, the flat output becomes two-dimensional as well.

The system is flat because all state variables and all system inputs can be expressed as a function of the flat output and a finite number of its derivatives. These conditions are checked by setting up the following two equations

$$
\begin{gathered}
x=\psi_{1}\left(y, \dot{y}, \ldots, y^{(\beta)}\right) \\
u=\psi_{2}\left(y, \dot{y}, \ldots, y^{(\beta+1)}\right)
\end{gathered}
$$

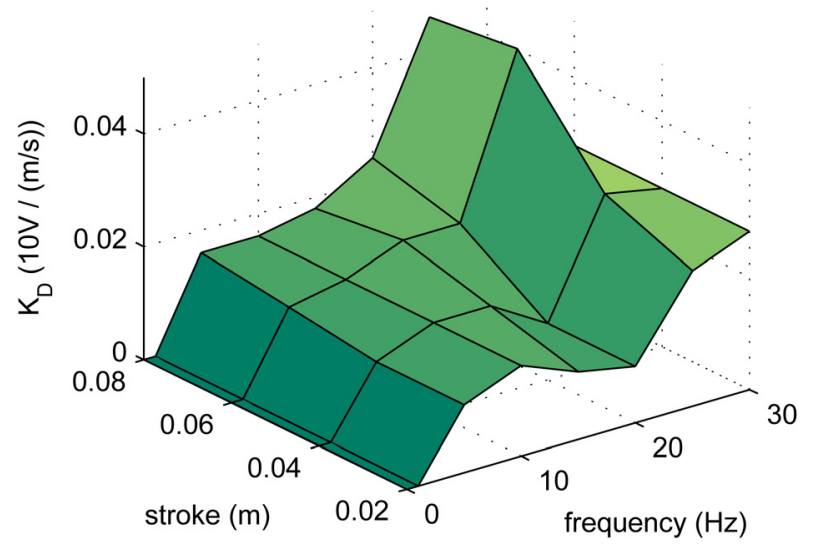

Fig. 7 Feedback controller variable gain $K_{D}$
In order to find the desired equation for the voltage $u_{1}$ as a function of the desired trajectory, these equations are combined and symbolically solved using a computer algebra system (CAS). The result is shown in Eq. (14). Following an analogous procedure for $x_{v} \leq 0$ leads to a similar result (Eq. (15))

$$
\begin{aligned}
& u_{1}=\left\{\begin{array}{ll}
\psi_{+}\left(y, \ldots y^{(n)}\right) & \text { if } \quad x_{v} \geq 0 \\
\psi_{-}\left(y, \ldots y^{(n)}\right) & \text { if } \quad x_{v}<0
\end{array} .\right. \\
& \psi_{+}=\frac{1}{k_{v}} \cdot \frac{1}{\alpha \cdot 2 \pi \cdot r_{v}} \sqrt{\frac{\rho_{\text {oil }}}{p_{0}-\frac{m_{p} \ddot{y}_{1}+d_{p} \dot{y}_{1}-y_{2}}{A_{p}}}} \cdot\left(\frac{V_{0}}{2 E_{\text {oil }}} \frac{m_{p} y_{1}^{(3)}+d_{p} \ddot{y}_{1}-\dot{y}_{2}}{A_{p}}\right. \\
& \left.+k_{l i p} \frac{m_{p} \ddot{y}_{1}+d_{p} \dot{y}_{1}-y_{2}}{A_{p}}+k_{l i v} \dot{y}_{1}+A_{p} \dot{y}_{1}\right)
\end{aligned}
$$

$$
\begin{aligned}
\psi_{-}= & \frac{1}{k_{v}} \cdot \frac{1}{\alpha \cdot 2 \pi \cdot r_{v}} \sqrt{\frac{\rho_{\text {oil }}}{p_{0}+\frac{m_{p} \ddot{y}_{1}+d_{p} \dot{y}_{1}-y_{2}}{A_{p}}}} \cdot\left(\frac{V_{0}}{2 E_{\text {oil }}} \frac{m_{p} y_{1}^{(3)}+d_{p} \ddot{y}_{1}-\dot{y}_{2}}{A_{p}}\right. \\
& +k_{l i p} \frac{m_{p} \ddot{y}_{1}+d_{p} \dot{y}_{1}-y_{2}}{A_{p}}+k_{l i v} \dot{y}_{1}+A_{p} \dot{y}_{1}
\end{aligned}
$$

Equations (14) and (15) represent the inverted system equations for the two separate ranges of $x_{v}$. In contrast, Eq. (13) is formally no inverse system in the desired manner, because in addition to the flat output $y$ and its derivatives, it requires the valve slide position $x_{v}$ as a variable on the right hand side. To solve this problem, the two cases have to be inverted separately and the cases are distinguished afterward. For the purpose of case distinction, the actual valve slide position is the most obvious criterion. An alternative that leads to more precise results is explained in the following. Both equations output the voltage $u_{1}$, which represents the set point for the valve slide position. A positive voltage leads to a positive valve slide position and vice versa. As $u_{1}$ is the variable being calculated by the equations, using itself to select the equation would lead to a circular reference. Analyzing the two equations (14) and (15) shows that the sign of $u_{1}$ only depends on the terms inside the brackets, which is the same in both cases. As a result, the sign of $u_{1}$ and with it the information, which of the two equations is used to calculate $u_{1}$, can be found by evaluating the term inside the brackets. The advantage of this more complex construction is that it takes into account the future (desired) valve position to select the correct model equation when calculating the future valve input voltage. This means that the calculation is always done for one consistent sampling point in time. As a result, discontinuities in the voltage signal are prevented.

The resulting algorithm outputs $u_{1}=\hat{u}_{v}(t)$, which is the desired voltage that a fictitious plant without dead time would need as an input in order to track the desired trajectory (see Fig. 4). In order to find $u_{v}(t)$, which is usable for the entire physical plant, the dead time element would have to be inverted as well. It is well known that the inversion of dead time elements cannot be realized, because the resulting inverse system would be acausal. Therefore, only the valve flow, the working piston, and the proportional part (respectively, the $P T_{4}$ part) of the valve dynamics are treated as shown above, but the dead time element is not inverted. Instead, the data fed to the inverse model's inputs are shifted in time. This means that the block calculating the voltage $u_{v}$ at time $t$ does not expect the desired plunger position $x_{1}$ at time $t$ at its input, but the desired plunger position at time $t+T_{d}$, which is in the future. The same demand applies for the load force. Thus, both the load force and the desired trajectory have to be known ahead of time. In case of the free piston engine test stand, this is possible, because the trajectory is periodic per definition and the forces can be predicted based on a model of the engine's components. 
Table 3 Methods for considering the error history in a feedback control signal

\begin{tabular}{lc}
\hline \hline Controller & Signal equation \\
\hline Integral & $e(t)+e\left(t-t_{s}\right)+e\left(t-2 t_{s}\right)+\cdots$ \\
Repetitive & $e(t)+e\left(t-t_{0}\right)+e\left(t-2 t_{0}\right)+\cdots$ \\
\hline \hline
\end{tabular}

\section{Feedback Control Design}

In order to compensate unknown disturbances and model uncertainties, a feedback controller is superposed. The more precisely the feedforward part works, the lower the absolute value of the control signal generated by the feedback part will be. Two feedback control concepts are investigated in the following.

The first implemented feedback controller is the well-known proportional-integral-derivative (PID) controller. It is a widespread algorithm that is being used to solve many hydraulic control problems, e.g., Ref. [13]. When manually tuning the parameters, it turns out that the best parameter set depends on the operating point of the working piston. For good performance, the controller parameters have to be set depending on the desired operating frequency ( $f_{0}$, equivalent to rpm in a crank-driven engine) and the stroke. This is why the PID controller is expanded to a variable gain PID algorithm, varying its parameters according to a characteristic diagram with the input variables frequency and stroke and the output variables proportional controller gain, integral controller gain, and derivative controller gain. The setup is found experimentally by running the systems in different operating points and tuning the controllers gains for lowest tracking error in every operating point. The result is one characteristic diagram for each of the three parameters $K_{P}$, $K_{I}$, and $K_{D}$ as shown in Figs. 5-7.

On the other hand, a repetitive PD controller is considered. Repetitive control $[13,14]$ is a control scheme which can be used in control loops in which the disturbances and/or the setpoint signals are dominantly periodic in time with trial length $1 / f_{0}$. These prerequisites are fulfilled for the system regarded here. On the one hand, during steady state operation, the setpoint signal for the piston position is perfectly periodic. Even in case of transient operation, load changes are performed slowly compared to the cycle duration of the engine. On the other hand, a significant portion of the external forces acting on the hydraulic actuator is periodic due to the fact that they depend on the periodic piston position.

In comparison to the PID controller, the integrating branch is now replaced by a repetitive compensator. As the integrator in the PID is used to compensate the steady-state-error in time domain, the repetitive compensator compensates the steady-state-error in "trial domain" [15]. To illustrate the difference between the two methods, the discrete equations for calculating the integral/repetitive portion of the control signal are shown in Table 3 . The equations show that the integral controller uses the entire history to compensate the steady-state-error, whereas the repetitive compensator regards only the current sample and the corresponding samples in previous trials.

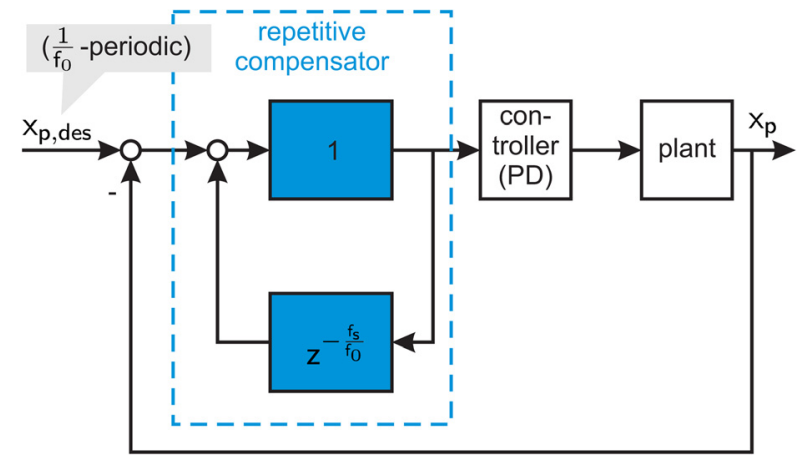

Fig. 8 Feedback control circuit with repetitive compensator

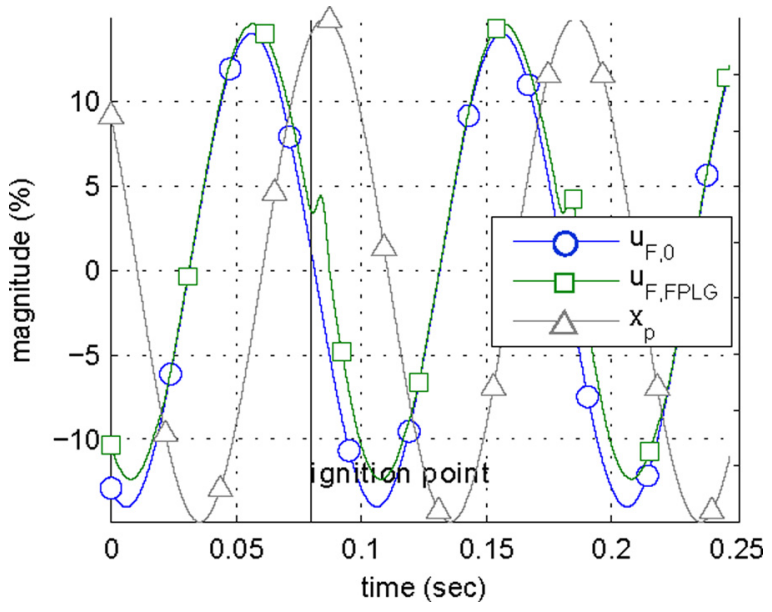

Fig. 9 Output signal of the feedforward controller without load $\left(u_{F, 0}\right)$ and with load $\left(u_{F F P L G}\right)$

A repetitive compensator can easily be realized using a delay element with a delay time $1 / f_{0}$ (equal to the trial length), which is trivial in a digital controller, see Fig. 8.

\section{Simulation and Measurement Results}

Figure 9 shows the control signal generated by the feedforward controller for a desired sine wave trajectory with a frequency of $10 \mathrm{~Hz}$ and a stroke of $90 \mathrm{~mm}$. First, the load forces are set to zero $\left(u_{F}, 0\right)$. Afterward, the load forces are generated by a simple combustion model $\left(u_{F, F P L G}\right)$. The combustion model (not subject to this paper) phenomenologically regards the release of energy and is similar to a Vibe function [16]. The peak around $t=0.08 \mathrm{~s}$ is calculated by the feedforward controller due to a sudden increase of combustion pressure after ignition. Although the peak in the figure looks comparatively small, it leads to large hydraulic forces. The additional signal adds an additional displacement to the valve slide, so that an additional amount of oil is pumped into or out of the chambers. Due to the fact that the piston trajectory remains unchanged, the additional amount of oil with high stiffness generates large forces ( $40 \mathrm{kN}$ in the shown example).

In order to validate the results of the inversion, the feedforward controller is used within a SIMULINK simulation. Therein, the model developed above is implemented as a plant. Also, the simplifications made prior to the inversion process have been implemented. As the plant is based on the same equations as the controller, a correct inversion will result in a perfect feedforward controller that tracks any trajectory without any deviation (as long as saturation does not become

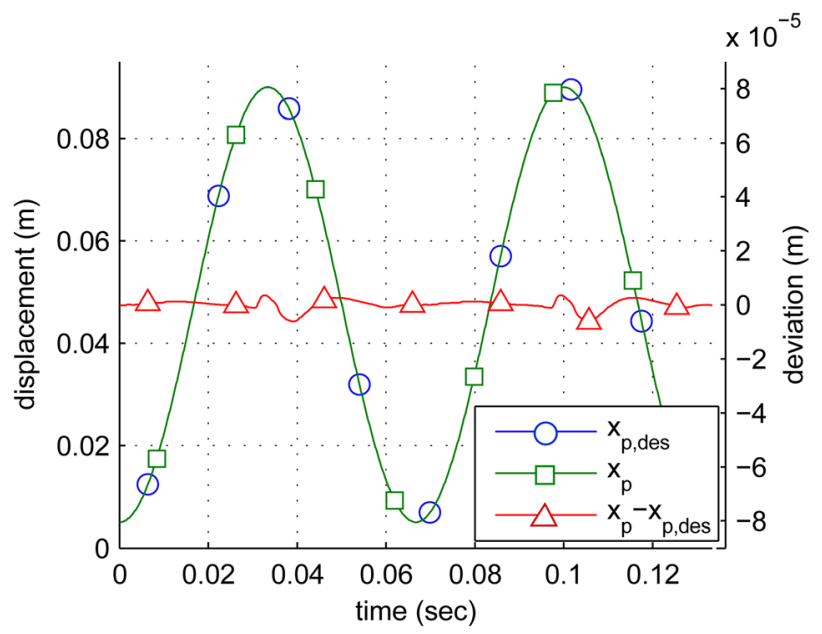

Fig. 10 Desired and actual trajectory in the simulation 


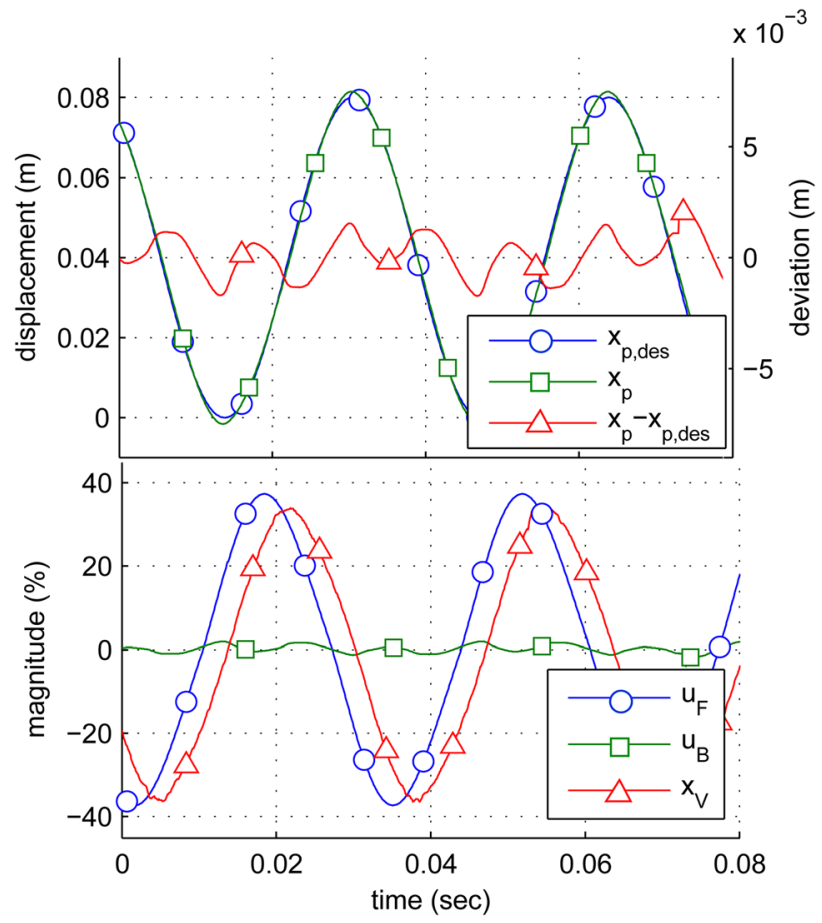

Fig. 11 Desired and actual trajectory at the test stand, actuating variable split up into feedforward and feedback portion (feedback controller: PID)

relevant). Indeed, the system can be operated and is drift free without adding a feedback circuit. The resulting deviation with a maximum below $10^{-5} \mathrm{~m}$ is minuscule (see Fig. 10). It can be further reduced by reducing the step size of the simulation. It can be assumed that the error converges toward zero for infinitesimal steps. In summary, the process of inversion including the treatment of discontinuity and dead time was successful.

Returning to the hardware test stand, model uncertainties and signal noise lead to a difference between the model and the actual behavior of the plant. A feedback controller is now required for compensation. In Fig. 11, the desired and the actual trajectory are compared at the operating point $30 \mathrm{~Hz} / 80 \mathrm{~mm}$. The deviation has a maximum of $1.9 \mathrm{~mm}$ and a root mean square of $0.8 \mathrm{~mm}$. In the lower plot of the Fig. 11, the control signal $u_{v}$ is shown and split up into the feedforward portion $u_{F}$ and the feedback portion $u_{B}$. Obviously, the feedforward controller works good enough to

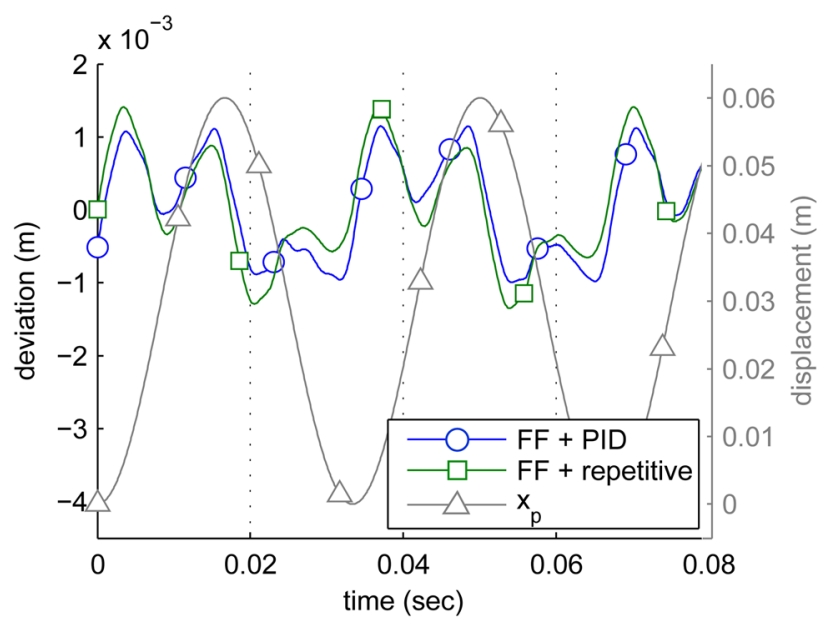

Fig. 12 Control deviation with conventional PID and PDrepetitive feedback controller $(30 \mathrm{~Hz} / 60 \mathrm{~mm})$ provide most of the output signal. The maximum of its output is 7.6 times the maximum of the feedback control signal.

Regarding different operating points, a general result is that the system is significantly more sensitive to changes in frequency than in stroke. Deviation increases rapidly for frequencies of 30 $\mathrm{Hz}$ or higher, whereas the relation between stroke and deviation is approximately linear. The highest frequency of $33 \mathrm{~Hz}$ was reached with the described setup.

Replacing the PID controller by a repetitive PD controller does not lead to significant improvements. Depending on the operating point, the entire system performs slightly better or slightly worse than the same system with a conventional PID controller (see Fig. 12). Because of the high calculating capacity, needed by the repetitive PD controller, the conventional PID controller becomes more attractive than the repetitive controller for the application discussed here.

The reason that makes the repetitive structure underachieve the expectations is again the dead time that dominates the plant's behavior. Simulations show that the proposed repetitive control structure performs poorly with non phase-minimal plants. This is due to the fact that in a control circuit with dead time $T_{d}$, the cause for a deviation at time $\mathrm{t}$ is at times $t-n / f_{0}-T_{d}$, whereas the proposed structure regards only times $t-n / f_{0}$ to compensate the deviation.

Concerning another aspect, the repetitive control structure is more fault-tolerant than expected. Running the system instationary in the sense of varying frequency or stroke during operation does not cause problems. Although this scenario does not comply with the requirement of a periodic reference signal, the algorithm adapts fast enough to fade from one operating point to any other within less than $5 \mathrm{~s}$, depending on how much the two operating points differ. Practically, the instationary behavior is as good as with the nonrepetitive PID controller. Obviously, the limiting factor for the rapidness of changes is not the controller structure but the capability of the hydraulic supply pump to provide rapidly changing massflows of oil.

\section{Conclusion}

A control concept for a hydraulic actuator with high demands concerning both stroke and frequency has been developed. For this purpose, a nonlinear model of the plant consisting of a hydraulic cylinder and a multistage servo valve was found and was inverted after some simplifications. Nonlinearity, discontinuity, and dead time contained in the plant were regarded when generating a feedforward control algorithm based on the inverse model. The feedforward controller calculates the optimal input signal for tracking a desired trajectory and takes into account the predicted load forces on the system. The validity of the implemented solution has been proven in simulations; the practical benefit has been verified experimentally. At the physical plant, a feedback controller is inevitably necessary. The performance of a repetitive controller was compared to the performance of a PID controller. Due to the non phase-minimal characteristics of the plant, the implemented repetitive controller does not lead to significant improvements compared to a conventional PID algorithm. In the most dynamic test runs, the maximum plunger velocity exceeded $8 \mathrm{~m} / \mathrm{s}$, which corresponds to an average velocity of $4 \mathrm{~m} / \mathrm{s}$. This is a significant expansion of the operating range of hydraulic actuators. The improved dynamic behavior of the hydraulic actuator allows for the development of free piston engines at higher frequencies and strokes. This is an important step for the development process of a prototype free piston linear generator.

\section{Nomenclature}

$A_{p}=$ effective area of hydraulic piston

$m_{p}=$ overall moving mass (plunger, piston, etc.)

$d_{p}=$ overall friction coefficient

$E_{\text {oil }}=$ elastic modulus of hydraulic fluid

$V_{0}=$ hydraulic volume in neutral position of plunger

$k_{\text {lip }}=$ coefficient of pressure proportional leakage 


$$
\begin{aligned}
k_{l i v} & =\text { coefficient of velocity proportional leakage } \\
r_{v} & =\text { radius of valve slide } \\
\alpha & =\text { orifice coefficient } \\
T_{d} & =\text { valve dead time } \\
x_{p}, \text { des } & =\text { plunger position-desired } \\
x_{p} & =\text { plunger position-measured } \\
x_{v} & =\text { main valve slide position } \\
e & =\text { control deviation } \\
F_{L o a d} & =\text { load force } \\
f_{0} & =\text { operating frequency FPLG } \\
f_{s} & =\text { sample frequency of the digital controller } \\
p_{0} & =\text { hydraulic supply pressure } \\
p_{A}, p_{B} & =\text { hydraulic pressure in actuator chamber A/B } \\
Q_{A}, \dot{Q}_{B} & =\text { total volume flow into chamber A/B } \\
\dot{Q}^{\prime} & =\text { total volume flow in reduced state system } \\
\dot{Q}_{0 \rightarrow A}, \dot{Q}_{0 \rightarrow B} & =\text { volume flow from pressure supply to chamber } \\
\dot{Q}_{A \rightarrow T}, \dot{Q}_{B \rightarrow T} & =\text { A/B } \\
u_{v} & =\text { colume flow from chamber A/B to tank } \\
\hat{u}_{v} & =\text { delayed control voltage of servo valve } \\
u_{F} & =\text { feedforward generated control voltage } \\
u_{B} & =\text { feedback generated control voltage } \\
x, x_{1}, \ldots, x_{4} & =\text { state space: state variables } \\
u, u_{1}, u_{2} & =\text { state space: input variables } \\
y & =\text { flat output }
\end{aligned}
$$

\section{References}

[1] Pohl, S.-E., 2007, "Der Freikolbenlineargenerator-Theroretische Betrachtungen des Gesamtsystems und experimentelle Untersuchungen zum Teilsystem der Gasfeder," Ph.D. thesis, Helmut-Schmidt-Universität, Hamburg, Germany.
[2] Deuschl, M., 2006, "Gestaltung eines Prüffelds für die Fahrwerksentwicklung unter Berücksichtigung der virtuellen Produktentwicklung," Ph.D. thesis, Technische Universität München, Munich, Germany.

[3] Crewe, A. J., and Severn, R. T., 2001, The European Collaborative Programme on Evaluating the Performance of Shaking Tables, Earthquake Engineering Research Centre, University of Bristol, UK.

[4] Murrenhoff, H., 2005, Grundlagen der Fluidtechnik—Teil 1: Hydraulik, Shaker Verlag, Aachen, Germany.

[5] Backé, W., 1986, Servohydraulik, Umdruck zur Vorlesung, Institut für hydraulische und pneumatische Antriebe und Steuerungen der RWTH Aachen, Aachen, Germany.

[6] Karpenko, M., and Sepehri, N., 2010, "On Quantitative Feedback Design for Robust Position Control of Hydraulic Actuators," Con. Eng. Pract., 18(3), pp. 289-299.

[7] Niksefat, N., Sepehri, N., and Wu, Q., 2006, "Design and Experimental Evaluation of a QFT Contact Task Controller for Electro-Hydraulic Actuators," Int. J. Robust Nonlinear Control, 17, pp. 225-250.

[8] Jones, D., and Mansoor, S., 2004, "Predictive Feedforward Control for a Hydroelectric Plant,” IEEE Trans. Control Syst. Technol., 12, pp. 956-965.

[9] Horn, J., Bamberger, J., Michau, P., and Pindl, S., 2003, "Flatness-Based Clutch Control for Automated Manual Transmissions," Control Eng. Pract., 11, pp. 1353-1359.

[10] Vossoughi, G., and Donath, M., 1995, "Dynamic Feedback Lineariszation for Electrohydraulic Actuated Control Systems,” J. Dyn. Syst. Meas. Control, 117, pp. $468-477$.

[11] Alleyne, A., 1996, "Nonlinear Force Control of an Electrohydraulic Actuator," Proceedings of Japan/USA Symposium on Flexible Automation, Boston.

[12] Fliess, M., Levine, J., Martin, P., and Rouchon, P., 1992, "On Differentially Flat Nonlinear Systems," Nonlinear Control System Design, Pergamon, Bordeaux, France, pp. 408-412.

[13] Plummer, A. R., 2007, "Control Techniques for Structural Testing: A Review," Proc. Inst. Mech. Eng., Part I: J. Syst. Control. Eng., 221, pp. 139-169.

[14] Moore, K. L., and Xu, J.-X., 2000, "Special Issue on Iterative Learning Control," Int. J. Control, 73, pp. 819-999.

[15] Dijkstra, B., 2004, "Iterative Learning Control, With Applications to a WaferStage," Ph.D. thesis, Delft University of Technology, Delft, Netherlands.

[16] Pischinger, R., Klell, M., and Sams, T., 2009, Thermodynamik der Verbrennungskraftmaschine, Springer, Wien, Austria. 